\title{
Audit of disposal of clinically confidential information
}

\author{
AIMS AND METHOD \\ We conducted a 3-cycle audit of \\ disposal of clinically sensitive \\ information in a mental health unit, \\ in order to identify and reduce \\ potential breaches in patients' \\ confidentiality. Material from waste \\ bins in administrative areas of a \\ mental health unit was examined \\ every evening during each period of \\ the audit.
}

\author{
RESULTS \\ The first search, conducted over a \\ 3-week period, yielded 11 documents \\ containing highly-sensitive \\ information about patients. After \\ feedback to staff and improvement \\ of shredding facilities, no sensitive \\ information was found during the \\ follow-up 3-week survey, 3 months \\ later. However, a third survey 2 years \\ later found 24 highly-sensitive items
}

\author{
after one week, despite shredding \\ facilities being maintained. \\ CLINICAL IMPLICATIONS \\ Changes in behaviour identified in \\ this audit appear to be due to \\ education rather than improved \\ facilities. All staff involved in patient \\ care need to maintain awareness of \\ the need for safe disposal of \\ confidential material.
}

Except under exceptional circumstances, clinicians have a duty to keep patient-related information confidential, especially outside the clinical team (Dolan, 2004; National Health Service, 2003). Failure to do so may result in internal disciplinary action or legal sanctions, as well as endangering doctor-patient relationships if unwitting disclosure comes to light. We became aware that some written material concerning patients was being discarded in waste bins. These bins were emptied daily into the hospital's general refuse system, creating a possibility that clinical information could reach unauthorised third parties. In order to identify the magnitude of this issue, we conducted an audit of unsatisfactory disposal of clinically sensitive documents relating to patients on a mental health unit.

\section{Methods}

We examined the contents of all waste-paper baskets and receptacles in the administrative areas of one mental health unit, which included seven clinical teams in general adult and old age services. Doctors' and secretaries' offices, seminar rooms, the photocopy machine room, library and corridor were searched each evening during the audit cycles. Colleagues were unaware that searches would take place. The gold standard for this audit was no pieces of confidential information being discarded.

All documents obtained from waste bins were examined, and those that were judged to contain clinical information on patients were rated blindly by two authors (Z.K. and J.W. or C.D. and J.W.). Material was categorised as 'sensitive' if it identified a patient and linked him/her to the mental health unit, but provided no other information. Material was regarded as 'very sensitive' if it identified a patient and contained details of history, diagnosis, treatment, Mental Health Act 1983 status, drug or alcohol use or other personal information.

Results of the initial survey were fed back to all relevant members of staff by a letter outlining the findings and a presentation at a staff academic meeting. As a result of the first audit cycle, a document shredder was moved from an office to a communal part of the unit and an additional confidential bin for safe disposal (by remote shredding) was installed. Follow-up surveys using the same methods were conducted at 3 months and 24 months after promulgation of the initial survey. The first two search cycles were 3 weeks long; the third was truncated to one week because of the amount of data amassed.

\section{Results}

Results of the three audit cycles are shown in Table 1. Some documents contained reference to more than one individual. In each case where multiple individuals were named, all items were judged to be extremely sensitive. The first search yielded 14 separate documents, 11 of which were judged to contain extremely sensitive clinical information. Three items that were recovered only gave names and dates of birth of patients. One set was torn in half, but was easily reassembled. No one clinical team or individual was identified as the source of the confidential material. After the initial audit and promulgation of the results, the second cycle found no confidential documents. However, a third cycle completed after a longer interval found a large number of highly-sensitive documents.

Examples identified during the three audits of 'highly sensitive' information linked to patients' names included:

- referral forms

- Mental Health Review Tribunal notification

- care programme approach care plans

- several partially completed psychiatric reports

- one complete psychiatric report

- psychiatric out-patient letters addressed to a general practitioner

- one set of 'handover' notes for a locum, listing 24 patient names, their diagnoses, treatment plans, medication and past history

\section{Discussion}

We were surprised about the large amounts of confidential material recovered from the first survey. The findings of 
Table 1. Numbers of sensitive and extremely sensitive documents recovered during each audit

\begin{tabular}{|c|c|c|c|c|c|}
\hline & Duration & 'Sensitive' & 'Extremely sensitive' & $\begin{array}{l}\text { Total documents } \\
\text { recovered }\end{array}$ & $\begin{array}{l}\text { Number of confidentiality } \\
\text { breaches* }\end{array}$ \\
\hline Search 1 October 2001 & 3 weeks & 3 & 11 & 14 & 34 \\
\hline Search 2 December 2001 & 3 weeks & 0 & 0 & 0 & 0 \\
\hline Search 3 December 2003 & 1 week & 7 & 24 & 31 & 47 \\
\hline
\end{tabular}

*Some documents included information on more than one patient.

this survey demonstrated the need for staff to be cautious with patient-sensitive material to ensure that confidentiality is not undermined. Consequently, we organised rapid and comprehensive dissemination of the results and discussed the findings with local managers, who arranged more accessible and less time-consuming shredding facilities. We felt reassured by the nil return of the second survey, and imputed this to be due to the provision of these facilities as much as to the increased awareness of staff about need to ensure confidentiality. The third audit had taken place after many of the staff had changed, including the appointment of two new consultants. The shredding facilities were still in place. The high return of the third search suggests that education, rather than facilities provided, is more important in affecting change of behaviour on this issue.

The documents found were distributed throughout the offices in the search area. It is worth noting that some bins offered up no documents, while others provided a daily supply. Particularly bountiful bins were in areas shared by several people and those found close to fax machines.

During this audit, we had concerns about entering offices without permission and having access to detailed confidential information. However, we feel that highlighting the extent of the problem and the need for intervention exceeded the potential confidentiality breach inherent in this study. Only clinicians were involved in the audit, and we kept our scrutiny of the material to a minimum. Once the information had been documented, it was shredded.

We recommend that in addition to easily-accessible shredders, mental health units institute regular education on the duty of confidentiality to all members of staff that have access to confidential patient information.

\section{Declaration of interest}

None.

\section{References}

DOLAN, B. (2004) Medical records. Disclosing confidential clinical information. Psychiatric Bulletin, 28,

NATIONAL HEALTH SERVICE (2003) Confidentiality: NHS Code of Practice. London: Department of Health: www.dh.gov.uk/asset Root/04/06/ 92/54/04069254.pdf

Craige Dietrich Senior House Officer in Psychiatry, Central and North West London Mental HealthTrust, Zahir Khan Senior House Officer in Psychiatry, Central and NorthWest London Mental HealthTrust, *James Warner Senior Lecturer/Consultant in Old Age Psychiatry, Imperial College London, Department of Psychiatry, Paterson Centre, 20 SouthWharf Road, LondonW2 1PD. E-mail: j.warner@imperial.ac.uk original papers 